A PRACTICAL NUMERICAL ALGORITHM TO COMPUTE STEADYSTATE GROUND LEVEL CONCENTRATION BY A $K$-MODEL

E. Runca

International Institute for Applied Systems Analysis, Laxenburg, Austria

RR-82-9

March 1982

Reprinted from Atmospheric Environment, volume 16 (1982)

INTERNATIONAL INSTITUTE FOR APPLIED SYSTEMS ANALYSIS

Laxenburg, Austria 
Research Reports, which record research conducted at IIASA, are independently reviewed before publication. However, the views and opinions they express are not necessarily those of the Institute or the National Member Organizations that support it.

Reprinted with permission from Atmospheric Environment 16:753-759, 1982.

Copyright (c) 1982 Pergamon Press Ltd.

All rights reserved. No part of this publication may be reproduced or transmitted in any form or by any means, electronic or mechanical, including photocopy, recording, or any information storage and retrieval system, without permission in writing from the copyright holder. 


\section{FOREWORD}

The International Institute for Applied Systems Analysis is conducting research on developing and verifying methods aimed at rationalizing and improving strategies for protecting the atmospheric environment. The work discussed in this paper was done as part of this program.

To assess the environmental impacts of potential emissions and of intervention strategies, an analyst must have models that simulate air pollution concentrations under the variety of conditions that may occur. The IIASA work on environmental quality and management explores the complexity, applicability, reliability, and suitability of such models, both for those currently in use and others that are proposed.

This paper introduces a new algorithm for computing ground-level concentrations of air pollutants under steady-state conditions. This new method is built on the classical advection-diffusion continuity equation (called the $K$-model in this paper); it improves the currently applied technique known as the Gaussian plume model.

JANUSZ KINDLER

Chairman

Resources and Environment Area 


\title{
A PRACTICAL NUMERICAL ALGORITHM TO COMPUTE STEADY-STATE GROUND LEVEL CONCENTRATION BY A $K$-MODEL
}

\author{
E. RUNCA \\ IIASA, Schloss Laxenburg, A-2361 Laxenburg, Austria \\ (First received 27 January 1981 and in final form 5 May 1981)
}

\begin{abstract}
A numerical algorithm to compute steady-state ground level concentration from elevated sources by means of a $K$-model which takes into account the spatial variability of wind and diffusivity and neglects horizontal diffusion is discussed. The boundary value problem to be treated, also for a point source, is always reduced to a two dimensional one and it is solved on an optimized grid. In this way the proposed method is made computationally comparable with the classical Gaussian plume model.
\end{abstract}

\section{INTRODUCTION}

The most common way to compute ground level concentration from an elevated point source is based on the application of the following formula known as the Gaussian plume model:

$$
\begin{aligned}
C(x, y, 0)= & \frac{Q}{\pi \bar{U} \sigma_{y}(x) \sigma_{z}(x)} \\
& \times \exp \left\{-\frac{y^{2}}{2\left[\sigma_{y}(x)\right]^{2}}-\frac{h^{2}}{2\left[\sigma_{z}(x)\right]^{2}}\right\} .
\end{aligned}
$$

In (1) the source is assumed to be located in the origin of the reference frame; the $x$-axis is chosen parallel to the wind direction; $h$ is the plume axis (i.e. the stack height plus the plume rise); $Q$ is the emission rate; $\bar{U}$ is a representative value of the wind speed (in general it is the value of the wind speed at the height of the chimney); $\sigma_{y}$ and $\sigma_{z}$ are the standard deviations of the concentration distribution in the $y$ and $z$ directions, respectively.

Equation (1) is obtained under the assumptions (see, e.g. Seinfeld, 1975) that the turbulent diffusion process is stationary and homogeneous; the emission rate is constant; the downwind diffusion is negligible compared with advection; the ground is a perfect reflector and the atmosphere is unbounded, i.e. no inversion is acting to suppress the vertical diffusion of the airborne matter, or at least, the ratio between the mixing height and the plume axis height is such that the influence of the inversion layer is felt quite far downwind from the source.

The Gaussian plume model was initially proposed by Sutton (1932), since then, in spite of the limiting assumption on which it is based, it has been extensively applied also to complex multiple source situations (see e.g. Turner, 1964, Shieh et al., 1972; Runca et al., 1976).

Another way to model dispersion of air pollutants is provided by the classical advection-diffusion equation which for a point source of constant emission rate $Q$, assuming steady-state, wind horizontally uniform, negligible horizontal diffusion, and the reference frame defined as for (1) takes the form:

$$
\begin{aligned}
U(z) \frac{\partial C}{\partial x}= & K_{y}(x, z) \frac{\partial^{2} C}{\partial y^{2}}+\frac{\partial}{\partial z}\left(K_{z}(x, z) \frac{\partial C}{\partial z}\right) \\
& +Q \delta(x) \delta(y) \delta(z-h)
\end{aligned}
$$

where $K_{y}$ and $K_{z}$ are the eddy diffusion coefficients (crosswind uniformity has been assumed) in the $y$ and $z$ directions, respectively, and $\delta(\cdot)$ is the Dirac's function. As for (1) the spatial resolution of (2) is limited by the Lagrangian length scale of the atmospheric turbulence. However, since (2) takes into account the spatial variation of wind and eddy diffusivity, it provides a more flexible model than the Gaussian one. Limitations of $K$-models have been discussed, among others, by Lamb and Seinfeld (1973) and Corrsin (1974).

The Gaussian formula has been generally preferred to the $K$-model as it avoids the costs and the problems connected with the use of (2); specifically when the effects of different meteorological conditions and different source heights on the ground level concentration (to which air quality standards apply) have to be analysed. With the Gaussian model, such analysis is computationally very simple, while the application of a $K$-model requires (the analytical solution, being in general, not available) the numerical integration of (2) on a tridimensional grid for each one of the considered cases. Hence, it appears that in order to make the $K$ model as usable as the Gaussian one the integration of (2) has to be made inexpensive in terms of both programing and computer time.

This paper presents a practical method to compute ground level concentration by a $K$-model. First, the numerical algorithm is discussed for the two dimensional case describing dispersion from a crosswind infinite line source. Then, the proposed method is tested for a situation having an analytical solution and thereafter, it is applied to neutral stability conditions. 
Finally, extension of the method to three dimensions is discussed

\section{TWO-DIMENSIONAL MODEL}

Assuming for the moment that the eddy diffusivity is only a function of the vertical coordinate, the concentration downwind from a line source is given by the solution to the following boundary value problem:

$$
\begin{array}{rlrl}
U(z) \frac{\partial C}{\partial x} & =\frac{\partial}{\partial z}\left(K_{z}(z) \frac{\partial C}{\partial z}\right)+\delta(x) \delta(z-h) \\
K_{z}(z) \frac{\partial C}{\partial z} & =0, & z & =0 \\
C(x, z) & =0, & & z=\infty \\
C(x, z) & =0, & & x<0 ; x=\infty .
\end{array}
$$

To generalize results given by the solution to (3) and related boundary conditions ( $3 \mathrm{a})-(3 \mathrm{c}), x, z, C, U$ and $K_{z}$ have been expressed in units of $\frac{H^{2} U(H)}{K_{z}(H)}, H$, $\frac{Q}{U(H) H}, U(H)$ and $K_{z}(H)$, respectively. In this way the source strength $Q$ [ see (3)] is normalized to one. $H$ is a suitable vertical length scale, hereafter, taken as the height of the planetary boundary layer.

For the boundary value problem $(3)-(3 c)$, the reciprocal theorem (Smith, 1957) gives:

$$
C_{h}(x, 0)=C_{0}(x, h)
$$

where $C_{h}(x, 0)$ is the ground concentration due to a source of height $h$ and $C_{0}(x, h)$ is the concentration at height $h$ due to a ground level source.

By virtue of the above equation, the solution to the boundary value problem (3)-(3c), with the source located at ground level $(h=0)$, allows one to derive the concentration at the ground for a source of any height Thus, as generally happens, if the objective is the computation of the concentration at ground level as a function of the source height, (3) needs to be integrated for a given meteorological condition only for the case $h=0$. This obviously results in quite substantial saving of computer time and poses the only problem of defining a numerical algorithm to provide accurate solution to (3) for the case $h=0$.

Definition of this numerical algorithm has to deal with the following problems: (i) approximation of the $\delta$-function representing the source term in (3) and (ii) approximation of the boundary condition ( $3 b)$.

Approximation of the $\delta$-function, as already proposed by Melli and Runca (1979), can be achieved by finding an approximate analytical solution to the boundary value problem (3)-(3c) in a region close to the source. Such an approximate solution is used to estimate the concentration profile in a downwind section (located at a suitable distance $x_{b}$ from the source) which is then taken as the left boundary of the integration region. Application of this procedure is discussed further later.

The definition of the upper boundary of the integration region is in some ways more complicated. Let us call $z_{s}$ the height of the upper boundary and for the moment, let us assume it constant. Since condition (3b) has to be approximately verified at every point downwind from the source, $z_{s}$ is determined by the right extreme (the farthest downwind point) of the integration region, as this is the point at which pollutant particles have spread to the maximum height. At the right extreme, the vertical concentration profile is quite smooth (assuming that this point is sufficiently far from the source) and can be described by a limited number of grid points uniformly spaced. Going backward towards the source, the concentration profile gets steeper and pollution is confined to layers close to the ground. To describe this situation, $z_{s}$ being constant, either the number of grid points has to be increased in the vertical sections close to the source or the grid points have to be unevenly spaced in such a way to have more points close to the ground where the concentration gradients are larger. Neither of these approaches is convenient. The increase of the grid points number means increase of memory and computer time. A non-uniform distribution of grid points can create, close to the source, large differences in the grid spacing and consequently, possible reduction of the accuracy of the numerical solution.

From the above considerations it seems more appropriate not to keep $z_{s}$ constant, but to consider it a function of the downwind distance from the source by defining it as the level at which in every section the concentration becomes negligible. With this choice, assuming that $z_{s}$ is known and considering, for the sake of simplicity, uniform spacing, both in the vertical and in the horizontal, the integration grid appears as in Fig. 1. Use of an upper boundary function of $x$ clearly allows one to describe accurately the concentration profile in every downwind section with the same number of points. This approach, as Fig. 1 illustrates, requires that the numerical integration be done on an irregular grid. However, we will show below that due to the type of the problem described by (3)-(3c), a standard finite difference scheme can be applied to the grid of Fig. 1.

\section{NUMERICAL ALGORITHM}

Considering the possibility of using disuniform grid spacing, both in the horizontal and in the vertical, the Crank-Nicolson scheme (see e.g. Richtmyer and Morton, 1967) has been applied to (3), yielding the finite difference equation:

$$
\begin{aligned}
C_{i, k}= & C_{i-1, k}+\frac{\Delta x_{i-1}}{\overline{U_{k}} \Delta z_{k}\left(\Delta z_{k-1}+\Delta z_{k}\right)} \\
& \left\{D\left[C_{i, k}\right]+D\left[C_{i-1, k}\right]\right\}
\end{aligned}
$$




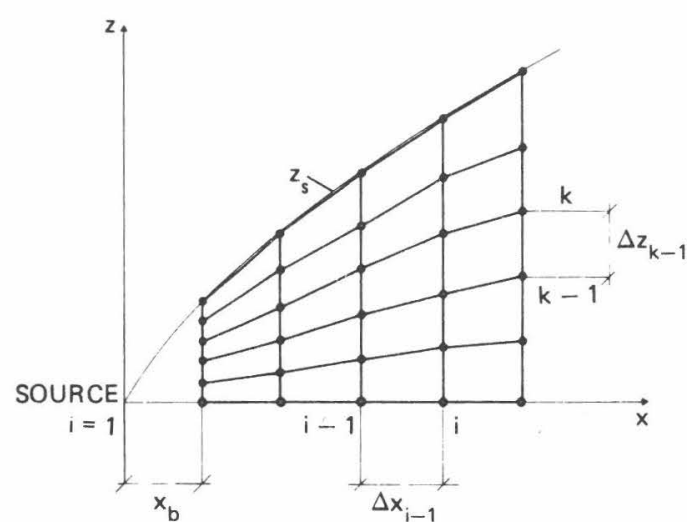

Fig. 1. Integration grid geometry with both horizontal and vertical uniform spacing. $z_{s}$ is the level at which the concentration becomes negligible (i.e. a very small fraction of the ground level concentration); $x_{b}$ is the location of the section where the vertical concentration profile is determined analytically.

with

and

$$
\bar{U}_{k}=\frac{2}{\Delta z_{k-1}+\Delta z_{k}} \int_{z_{k-1}+\frac{\Delta z_{k-1}}{2}}^{z_{k}+\frac{\Delta z_{k}}{\overline{2}}} U(z) \mathrm{d} z
$$

$$
\begin{aligned}
D\left[C_{k}\right]= & K_{k+\frac{1}{2}} C_{k+1}-\left(K_{k+\frac{1}{2}}+\frac{\Delta z_{k}}{\Delta z_{k-1}} K_{k-\frac{1}{2}}\right) C_{k} \\
& +\frac{\Delta z_{k}}{\Delta z_{k-1}} K_{k-\frac{1}{2}} C_{k-1} .
\end{aligned}
$$

In (4) $\Delta x_{i-1}$ is the horizontal interval between points $i-1$ and $i, \Delta z_{k}$ is the vertical interval between points $k$ and $k+1$, and $N$ is the number of points in every section.

The immediate consideration arising from the analysis of (4) is that, in order to compute the concentration in the $i$ th section, the concentration values in the $(i-1)$ th section have to be known at the same levels of the grid points of the $i$ th section. This is obviously not the case for the grid reported in Fig. 1. However, assuming that the concentration is known at the $(i-1)$ th section, the concentration values at the levels corresponding to the points of $i$ th section can be determined by an interpolation algorithm. Due to the definition of $z_{s}$ to the concentration in the points falling at or above $z_{s}$ is assigned the value zero. The situation is illustrated in Fig. 2.

In the application of this algorithm the condition to be fulfilled in every section is:

$$
\int_{0}^{z_{s}(x)} U(z) C(x, z) \mathrm{d} z=1 .
$$

The finite difference analog to (5) has the form:

$$
\sum_{k=1}^{N} U_{k} C_{i, k} \frac{\Delta z_{k-1}+\Delta z_{k}}{2}=1
$$

Equation (6) must not be violated when the profile

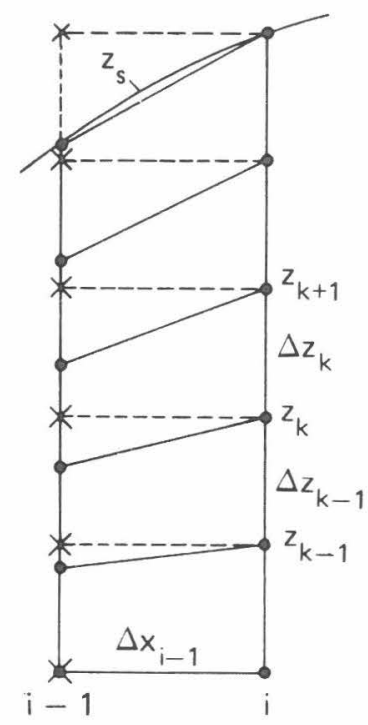

Fig. 2. Illustration of the application of the finite difference (4) (see text) to the grid of Fig. $1 . \times$ indicates the points of section $(i-1)$ corresponding to the points of section $i$. At these points the concentration is estimated by a linear interpolation.

concentration in the $(i-1)$ th section is described by the interpolated points. This implies (see Fig. 2) that accuracy is not lost when the grid spacing is changed in $(i-1)$ th section from the value $(\Delta z)_{i-1}=\frac{z_{s}\left(x_{i-1}\right)}{N-1}$ to the value $(\Delta z)_{i}=\frac{z_{s}\left(x_{i}\right)}{N-1}$. In other words, the number of grid points $N$ must be chosen in such a way that in the $(i-1)$ th section the true concentration profile can be approximated by a piecewise linear function described by $N$ values distributed once on the interval $0-z_{s}\left(x_{i-1}\right)$ and once on the interval $0-z_{s}\left(x_{i}\right)$. Achievement of this condition, given $N$, depends on $\Delta x_{i-1}$ and on $\frac{\mathrm{d} z_{s}}{\mathrm{~d} x}$. Since the highest growth rate of $z_{s}$ occurs close to the source, small values of $\Delta x$ are required close to the source. Thus the use of uniform horizontal grid spacing is not very convenient as it can require a very large number of points to describe the integration region. A horizontal grid spacing increasing with the distance from the source is then more suitable, as it will be shown later to the application of the numerical procedure described above.

Considering that the largest concentration gradient occurs at the ground, it seems convenient to use, also in the vertical, not a uniform grid spacing; but specifically a vertical grid size increasing with the distance from the ground. However, in the tests performed, a variable vertical grid spacing did not give the same increase in accuracy as the use of variable horizontal grid spacing. 
The improvement due to a more appropriate distribution of the points in the vertical was probably counterbalanced by the decrease of the order of the truncation error of the Crank-Nicolson scheme from $\Delta z^{2}$ to $\Delta z$, which occurs when disuniform spacing is used.

Up to now the choice of $N$ and its relation with $z_{s}$ has not been explained. Analysis of (4) indicates that concentration in the $i$ th section depends only on the $(i-1)$ th section. Thus, the "key-section" in the application of this algorithm is the one at $x=x_{b}$. Assuming that $z_{s}$ is known, $N$ must be such that the concentration profile can be described by a piecewise linear function in this section.

Estimation of the concentration profile at $x=x_{b}$ implies the approximation of the source term of (3). This is discussed below.

\subsection{Source term approximation}

The simplest way to approximate the source term is the replacement of the $\delta$-function with a step function in such a way that (6) is satisfied. This approach for a source located at ground introduces very large errors as the wind speed is zero at the source level. In addition, the representation of the $\delta$-function by a step function implies that some diffusion of the pollutant matter has occurred. Thus the step function has to be located at some undefined downwind distance. To give a better representation of the $\delta$-function term of (3), the method proposed by Melli and Runca (1979) can be used.

This method is based on the concept that in the region close to the source diffusion of pollutants depends substantially on wind and diffusivity values close to the source. This suggests replacing the wind and diffusivity with approximating functions which maintain the basic characteristics of the wind and diffusivity close to the source but allow at the same time the derivation of an analytical solution to (3). Such analytical solution can then be assumed as an approximation of the true concentration distribution in the region close to the source and used to compute the concentration profile at $x=x_{b}$. The analytical solution at $x=x_{b}$ is then approximated by a piecewise linear function over $N$ points chosen in such a way that (6) is satisfied. $z_{s}\left(x_{b}\right)$ is the chosen level at which the concentration is approximately zero. Computation of $z_{s}$ is discussed below.

\subsection{Definition of the upper boundary}

Once $z_{s}\left(x_{b}\right)$ is defined by taking it as the level at which the concentration is approximately zero, the ratio $\gamma=C_{z_{s}} / C_{z=0}$ is known at section $x=x_{b}$. The profile $z_{s}$ can then be computed under the assumption that $\gamma$ be the same in every section. Computation of $z_{s}$ is obviously trivial should the problem (3)-(3c) have an analytical solution. In the general case $z_{s}$ has to be determined by means of some approximate solution which guarantees an overestimate of it, as it is shown in the example reported below.

Verification of the method in a case for which the boundary value problem (3)-(3c) has an analytical solution as well as application to a situation representative of neutral atmospheric stability is now discussed.

\section{VERIFICATION AND APPLICATION}

For wind and diffusivity expressed by $U=z^{\alpha}$ and $K_{z}=z^{\beta}$, respectively, the solution to the boundary value problem (3)-(3c), with $h=0$, is (see Smith, 1957):

$$
\begin{aligned}
C_{0}(x, z)= & \frac{\alpha-\beta+2}{\Gamma(s)}\left[\frac{1}{(\alpha-\beta+2)^{2} x}\right]^{s} \\
& \exp \left[-\frac{z^{\alpha-\beta+2}}{(\alpha-\beta+2)^{2} x}\right]
\end{aligned}
$$

where $s=(\alpha+1) /(\alpha-\beta+2)$ and $\alpha-\beta+2>0$.

Defining $z_{s}$ as the height at which $C_{0}\left(x, z_{s}\right)$ $=\gamma C_{0}(x, 0)$ equation (7) gives:

$$
z_{s}=\left[(\alpha-\beta+2)^{2} x \log \frac{1}{\gamma}\right]^{\bar{\alpha}-\frac{1}{\beta}+\bar{z}} .
$$

With the above defined $z_{s}$, first, (3) has been integrated in the rectangular region $\left[0 \leqslant x \leqslant 0.15,0 \leqslant z \leqslant z_{s}(x\right.$ $=0.15)]$ by distributing uniformly $M$ points in the horizontal and $N$ points in the vertical. Successively it has been integrated over the same number of points allowing $z_{s}$ to change with $x$ according to (8). In this computation $\Delta z$ changes with $x$ and is kept uniform in every vertical section (see Fig. 1). Finally, (3) has been integrated over the same number of points by taking $z_{s}$ function of $x$ and by using both in the horizontal and in the vertical a variable grid spacing.

The computations utilized for Figs 3 and 4, which will be illustrated below, refer to $\alpha=0.15$ and $\beta=1$

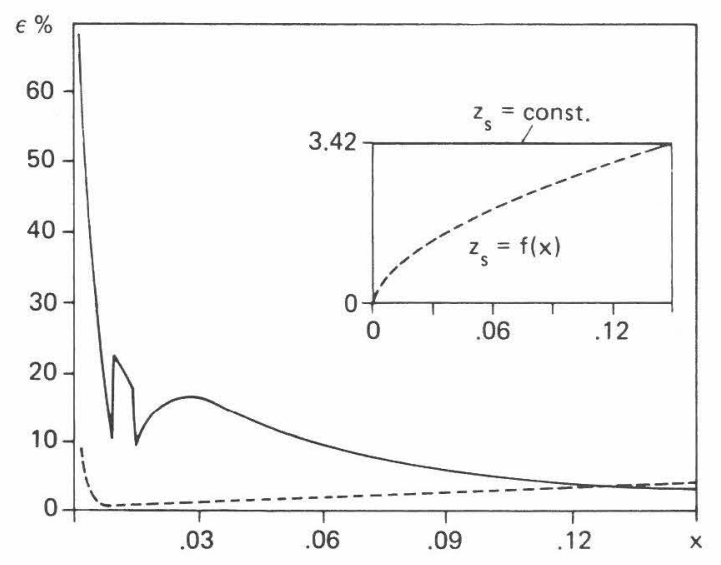

Fig. 3. Comparison of analytical and numerical solution. The plots represent the per cent error recorded in every vertical section in the location where the maximum absolute error occurred. The solid line refers to a constant upper boundary, the dashed one to an upper boundary function of the downwind distance (i.e. to a grid geometry as in Fig. 1). 


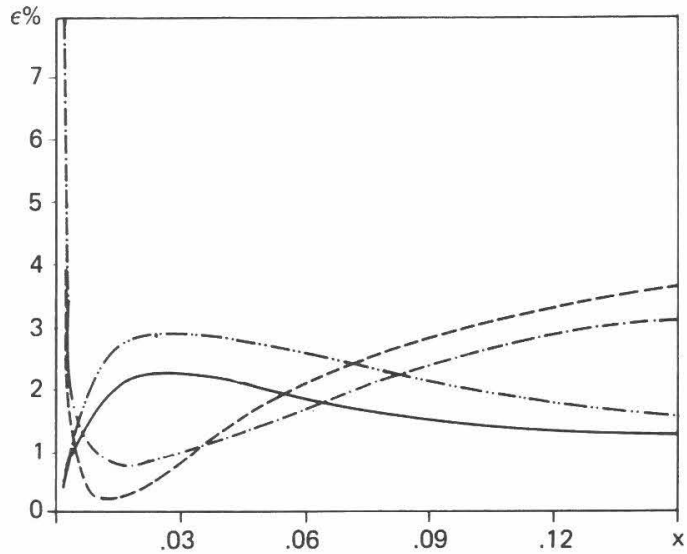

Fig. 4. Comparison of analytical and numerical solution. The plots represent the percentage error as defined in Fig. 3 for an upper boundary function of the downwind distance. The different lines refer to: ---- $\Delta x$ and $\Delta z$ uniform (same as in Fig. 3); -...- $\Delta x$ uniform and $\Delta z=f(z)$ $\ldots . .-\Delta x=f(x)$ and $\Delta z$ uniform; $-\Delta x=f(x)$ and $\Delta z$ $=f(z)$.

and were done with $M=151$ and $N=121$. Equation (7) provided the concentration profile at $x=x_{b}$, which was taken equal to $\Delta x_{1}$. In defining the geometry of the integration grid $N$ must be chosen in such a way that the constant flux condition, (6), is verified also by the concentration profile obtained after the application of the interpolation procedure. ${ }^{*}$ This implies that $N$ must be sufficiently large. In the tests performed, (6) was always accurately approximated at 121 points. Satisfactory approximation was also achieved at 80 points. However, results here are reported for $N=121$ in order to show that even in the case of a relatively refined uniform grid an upper variable boundary produces a remarkable increase in the accuracy of the numerical solution. Such an increase is more pronounced with coarse grids.

Figure 3 displays the per cent error computed in every vertical section by comparison with the analytical solution, (7), at the point where the maximum absolute error was found. The error is reported both for $z_{s}=$ const and $z_{s}=f(x)$ as given by (8) with $\gamma$ $=10^{-9}$. Use of $z_{s}$ function of $x$ reduces the error of one order of magnitude. Further reduction of the error is achieved by taking $\Delta x=f(x)$ and $\Delta z=f(z)$ as shown in Fig. 4. The plots of Fig. 4 were obtained by defining both $\Delta x_{1}$ and $\Delta z_{i, 1}$ equal to one-tenth of their respective values corresponding to the uniform grid spacing distribution. The successive values of $\Delta x$ and $\Delta z_{i}$ were then increased in such a way to reach with 150 and 120 intervals respectively the horizontal coordinate $x=0.15$ and the vertical coordinate $z_{i}=z_{s}\left(x_{i}\right)$. The plot of Fig. 4 indicates that the accuracy of the results depends strongly on the geometry of the grid. However, this point is here not further investigated. More relevant is the application of the proposed

* Rescaling of the concentration values, in order to verify (6), is only allowed at $x=x_{b}$. algorithm to the general case in which the analytical solution to the boundary value problem (3)-(3c) is unknown. This is described in the following with reference to dispersion in a neutral atmosphere.

Both theoretical (see, e.g. Shir, 1973; Wyngaard et al., 1974) and experimental work (Robins, 1978) have shown that the neutral vertical eddy diffusivity profile can be represented by an exponential law, which, following Shir and Shieh (1974) can be expressed in normalized units by the function:

$$
K_{z}=z \mathrm{e}^{-\rho(z-1)} .
$$

In (9) $\rho$ is a dimensionless parameter (approx. equal to 4 for neutral conditions) whose reciprocal gives the fraction of the height of the planetary boundary layer at which the maximum value of $K_{z}$ occurs.

With $U=z^{\alpha}$ and $K_{z}$ given by (9), (3) takes the form:

$$
z^{\alpha} \frac{\partial C}{\partial x}=\frac{\partial}{\partial z}\left(z \mathrm{e}^{-\rho(z-1)} \frac{\partial C}{\partial z}\right)+\delta(x) \delta(z) .
$$

Straightforward analysis of (10) shows that the concentration close to the source can be approximated by the solution to the equation:

$$
z^{\alpha} \frac{\partial C}{\partial x}=\frac{\partial}{\partial z}\left(z \mathrm{e}^{\rho} \frac{\partial C}{\partial z}\right)+\delta(x) \delta(z)
$$

obtained by replacing in (10) the vertical eddy diffusivity profile with its tangent in the origin. Considering the scaling factor $\mathrm{e}^{\rho}$ and noticing that $\beta=1$ the solution to (11) is derived from equation (7) in the form:

$$
C=\frac{\mathrm{e}^{-\rho}}{(1+\alpha) x} \exp \left[-\frac{z^{1+\alpha} \mathrm{e}^{-\rho}}{(1+\alpha)^{2} x}\right] .
$$

Equation (12) provides the required approximation of the concentration profile at $x=x_{b}$. In this way no arbitrary approximation of the source term has to be done for the application of the finite difference (4).

Equation (12) can be also used to provide the estimation of the upper boundary $z_{s}$. This stems immediately from the comparison of (10) with (11). Being $K_{z}=z \exp (\rho)$ greater than $K_{z}=z \exp [-z(\rho$ $-1)]$ at any level, (11) describes a process in which the material diffuses faster, and therefore to higher levels, than in the situation described by (10). Hence (12) guarantees an overestimate of $z_{s}$.

It is expected that, in the majority of the cases, it should be possible, by proceeding in a way similar to the example discussed above, to determine for the boundary value problem (3)-(3c) both an approximation of the concentration in the region close to the source and an estimate of the upper boundary $z_{s}(x)$ of the integration region. With this assumption the method acquires a general applicability. Its computational efficiency is apparent in a multiple source situation. In fact, said $\left(x_{k}, h_{k}\right)$ the location and the effective height of the $k$ th-source, respectively, the concentration at the ground due to $N_{s}$-sources is simply given, for a given meteorological condition, by: 


$$
C(x, 0)=\sum_{k=1}^{N_{s}} C_{0}\left(x-x_{k}, h_{k}\right)
$$

with $C_{0}\left(x-x_{k}, h_{k}\right)=0$ for $\left(x-x_{k}\right) \leqslant 0$.

In (13) the concentration $C_{0}$ is the matrix given by the numerical integration of (3) with $h=0$. Simple interpolation procedures are used for the $\left(x-x_{k}, h_{k}\right)$ locations which do not follow in the points of the grid.

Since the reciprocal theorem proved by Smith (1957) does not depend on the functional form of the diffusion coefficients the proposed method and the related (13) hold also for diffusivity profiles which are a function of the downwind distance from the source. However, for the sake of completeness, we recall that if the diffusivity profile can be expressed as:

$$
K_{z}=f(x) g(z)
$$

the definition of the new variable (see also Csanady, 1973):

$$
\xi=\int_{0}^{x} f\left(x^{\prime}\right) \mathrm{d} x^{\prime}
$$

reduces the problem to the one described by (3)-(3c), in which $x$ and $K_{z}$ are respectively replaced by $\xi$ and $g(z)$.

\section{THREE-DIMENSIONAL MODEL}

Extension of the proposed method to the three dimensional situation described by (2) with boundary conditions similar to the ones given by the relations (3a)-(3c) has no specific limitation. The equivalent of (13) is:

$$
C(x, y, 0)=\sum_{k=1}^{N_{s}} C_{0}\left(x-x_{k}, y-y_{k}, h_{k}\right) .
$$

However, two difficulties arise. The first one concerns the loss of computational efficiency with respect to the two dimensional case. The second one derives from the fact that for (2), even in the case of $U, K_{y}$ and $K_{z}$ expressed by power law of the vertical coordinate, an analytical solution is generally not available, thus making problematic both the approximation of the source term and the estimation of $z_{s}$.

Both the problems mentioned above can be solved if it is assumed that the concentration profile in the $y$ direction is gaussian. This assumption which was proposed by Smith (1957) (see also Demuth and Berger, 1977) is suggested by the way in which $y$ variations appear in (2). There are also experimental evidences that the crosswind concentration distribution is approximately Gaussian. On this basis the solution to (2) for a ground level source can be assumed to have the form:

$$
C_{0}(x, y, z)=\chi_{0}(x, z) \frac{\mathrm{e}^{-\frac{y^{2}}{2\left[\sigma_{y}(x, z)\right]^{2}}}}{\sqrt{2 \pi} \sigma_{y}(x, z)} .
$$

Defining the following momentums of the con- centration distribution:

$$
C_{00}=\int_{-\infty}^{+\infty} C_{0} \mathrm{~d} y \text { and } C_{02}=\int_{-\infty}^{+\infty} y^{2} C_{0} \mathrm{~d} y,
$$

it is immediately seen that $C_{00} \equiv \chi_{0}(x, z)$ is the solution to the two dimensional boundary-value problem (3)-(3c) while

$$
\sigma_{y}(x, z)=\left(\frac{C_{02}}{C_{00}}\right)^{\frac{1}{2}}
$$

Use of (17) reduces the tri-dimensional problem to the two dimensional one. Equation (16) can be replaced by the most convenient expression:

$$
\begin{aligned}
C(x, y, 0)= & \sum_{k=1}^{N_{s}} \chi_{0}\left(x-x_{k}, h_{k}\right) \\
& \times \frac{\mathrm{e}^{-\frac{\left(y-y_{k}\right)^{2}}{2\left[\sigma_{y}\left(x-x_{k}, h_{k}\right)\right]^{2}}}}{\sqrt{2 \pi} \sigma_{y}\left(x-x_{k}, h_{k}\right)} .
\end{aligned}
$$

However, use of (19) implies the knowledge of $\sigma_{y}(x, z)$, which is given by (18). Hence, $C_{02}$ has to be determined; this involves the solution of the following boundary value problem:

$$
\begin{gathered}
U \frac{\partial C_{02}}{\partial x}=\frac{\partial}{\partial z}\left(K_{z} \frac{\partial C_{02}}{\partial z}\right)+2 K_{y} C_{00} \\
K_{z} \frac{\partial C_{02}}{\partial z}=0, \quad z=0 \\
C_{02}(x, z)=0, \quad z=\infty \\
C_{02}(x, z)=0, \quad x=0 ; x=\infty .
\end{gathered}
$$

In deriving (20) use has been made of the Dirac's function property:

$$
\int_{-\infty}^{+\infty} f(y) \delta\left(y-y_{0}\right) \mathrm{d} y=f\left(y_{0}\right) .
$$

The solution of (20) with the related boundary conditions presents no difficulty. The same grid adopted for computing $C_{00}$ and a modified (4) to take into account the forcing term $\left(2 K_{y} C_{00}\right)$ can be used to integrate the boundary value problem $(20)-(20 c)$. Large errors occur at the upper boundary $z_{s}$ where both $C_{00}$ and $C_{02}$ go to zero. The evaluation of $\sigma_{y}$ cannot therefore be extended up to $z_{s}$; the computation must terminate a few grid points below $z_{s}$.

With the above formulation, the tri-dimensional case is reduced to the solution of two bi-dimensional problems. For the sake of completeness, it must be added that for the special case of lateral diffusivity having the form

$$
K_{y}=f(x) U(z)
$$


(20) has the simple solution

$$
C_{02}=2 C_{00} \int_{0}^{x} f\left(x^{\prime}\right) \mathrm{d} x^{\prime} .
$$

Thus, for those circumstances in which (21) holds the tri-dimensional problem is computationally equivalent to the bi-dimensional one.

\section{CONCLUSION}

Use of the reciprocal theorem proved by Smith (1957) and the definition of a simple finite difference algorithm have made possible the computation of steady-state ground level concentration downwind of both single and multiple source situations by a $K$ model without any loss of computational efficiency in comparison with the classical Gaussian plume model.

The proposed method requires also in a point source situation the solution of only bi-dimensional boundary value problems. It can therefore be programmed on a very small computer and is suitable to interactive languages, in this way providing the user with the capability to analyse in a very straightforward manner concentration profiles due to different source distributions as well as effects of grid geometry and parameters on the solution.

It is well known that $K$-theory provides only an approximate description of the processes which affect atmospheric diffusion. For those situations in which $K$-theory can be applied the proposed method can replace the Gaussian plume model. At more or less the same cost it provides the user with the possibility to analyse the effect on the steady-state ground level concentration of wind and diffusivity spatial variability, both in single and multiple source situations.

\section{REFERENCES}

Corrsin S. (1974) Limitations of gradient transport models in random walks and in turbulence. Adv. Geophys. 18A, 25-60.

Csanady G. T. (1973) Turbulent Diffusion in the Environment D. Reidel Publishing Company, Dordrecht-Holland.

Demuth C. and Berger A. (1977) Evaluation de la pollution à l'aide d'un modèle à coefficient de diffusion variables. Scientific Report AL/1/4.1.5, National R-D Program on Environment-Air, Services for Science Policy Programming, Belgium.

Lamb R. G. and Seinfeld J. H. (1973) Mathematical modeling of urban air pollution. Envir. Sci. Technol. 7, 253-261.

Melli P. and Runca E. (1979) Gaussian plume model parameters for ground-level and elevated sources derived from the atmospheric diffusion equation in a neutral case, J. appl. Met. 18, 1216-1221.

Richtmyer R. D. and Morton K. W. (1967) Difference Methods for Initial-Value Problems. Interscience, New York.

Robins A. G. (1978) Plume dispersion from ground level sources in simulated atmospheric boundary layers. Atmospheric Environment 12, 1021-1032.

Runca E., Melli P. and Zannetti P. (1976) Computation of long-term average $\mathrm{SO}_{2}$ concentration in the Venetian area. Appl. Math. Mod. 1, 9-15.

Seinfeld J. H. (1975) Air Pollution: Physical and Chemical Fundamentals. McGraw-Hill, New York.

Shieh L. H., Halpern P. K., Clemens B. A., Wang H. H. and Abraham F. F. (1972) Air quality diffusion model: application to New York City. IBM J. Res. Dev. 16, 162-170.

Shir C. C. (1973) A preliminary numerical study of atmospheric turbulent flows in the idealized planetary boundary layer. J. atmos. Sci. 30, 1327-1339.

Shir C. C. and Shieh L. H. (1974) A generalized urban air pollution model and its application to the study of $\mathrm{SO}_{2}$ distributions in the St. Louis Metropolitan Area. J. appl. Met. 13, 185-204.

Smith F. B. (1957) The diffusion of smoke from a continuous elevated point source into a turbulent atmosphere. J. Fluid. Mech. 2, 49-76.

Sutton O. G. (1932) A theory of eddy diffusion in the atmosphere. Proc. R. Soc. Lond. 135A, 143-165.

Turner D. B. (1964) A diffusion model for an urban area. $J$. appl. Met. 3, 83-91.

Wyngaard J. C., Coté O. R. and Rao K. S. (1974) Modeling the atmospheric boundary layer. Adv. Geophys. 18A, 193-212. 


\section{RELATED IIASA PUBLICATIONS}

\section{RESEARCH REPORTS}

The Smeared Concentration Approximation Method: A Simplified R.L. Dennis

Assessment of Alternative Energy/Environment Futures for Austria

1977-2015

W.K. Foell, R.L. Dennis, M.E. Hanson, L.A. Hervey,

A.H. Hoelzl, J.P. Peerenboom, E. Poenitz

Real-Time Forecasting of Air Pollution Episodes in the Venetian

Region. Part 1: The Advection-Diffusion Model

E. Runca, P. Melli, A. Spirito

Part II: The Kalman Predictor

G. Fronza, A. Spirito, A. Tonielli

Real-time Control of Sulphur Dioxide Emissions from an Industrial Area

P. Melli, P. Bolzern, G. Fronza, A. Spirito

Reprinted from Atmospheric Environment, Vol. 15, 1981 pp. 653-666

Validation and Physical Parametrization of a Gaussian Climatological Model Applied to a Complex Site

E. Runca, A. Longhetto, G. Bonino

Reprinted from Atmospheric Environment, Vol. 16(2), 1982

pp. 259-266

\section{COLLABORATIVE PAPERS}

Statistical Analysis of Winter Sulphur Dioxide Concentration Data in Vienna

P. Bolzern, G. Fronza, C. Überhuber

An Analysis of Finite Difference and Galerkin Techniques applied to the Simulation and Diffusion of Air Pollutants from a Line

Source

E. Runca, P. Melli, F. Sardei

$\begin{array}{ll}\text { CP-81-25 } & \begin{array}{l}\text { Single } \\ \text { copies } \\ \text { free }\end{array} \\ \text { CP-81-26 } & \begin{array}{l}\text { Single } \\ \text { copies } \\ \text { free }\end{array}\end{array}$

\section{WORKING PAPER}

Crop Yields in $\mathrm{CO}_{2}$-enriched Atmosphere

P. Kauppi 

